\title{
МЕТОДИКА ОЦЕНКИ СОВМЕСТИМОСТИ НАУЧНО-ИССЛЕДОВАТЕЛЬСКОГО КОЛЛЕКТИВА
}

\author{
(C) 2020 Седякина Анна Александровна \\ Высшая школа сервиса и торговли \\ Санкт-Петербургский политехнический университет Петра Великого (СПбПУ), \\ Россия, Санкт-Петербург \\ E-mail: aasedyakina@mail.ru \\ (C) 2020 Конников Евгений Александрович \\ кандидат экономических наук, Высшая инженерно-экономическая школа \\ Санкт-Петербургский политехнический университет Петра Великого (СПбПУ), \\ Россия, Санкт-Петербург \\ E-mail: konnikov.evgeniy@gmail.com
}

Процесс перехода к цифровой экономике определяет трансформацию всех общественных институтов и сфер жизнедеятельности. Данный процесс неизбежно затрагивает сферу науки и высшего образования, в которой все большее значение приобретают междисциплинарные исследования, а также мобильность и масштабируемость научно-исследовательских коллективов. На данный момент перед менеджментом научно-исследовательских и образовательных организаций стоит задача формирования универсальной инновационной среды, в которой в зависимости от поставленной задачи, может в кратчайшие сроки быть сформирован эффективный научно-исследовательский коллектив. Процесс формирования подобных коллективов является крайне комплексным и должен учитывать множество факторов, так как помимо профессиональных компетенций участников коллектива, необходимо прогнозировать специфику процесса их коммуникации. Однако, в условиях развития информационной среды значительно возрастает массив аналитической информации, что позволяет моделировать процесс коммуникации потенциальных участников научноисследовательского коллектива. В рамках данной статьи проводится методика, позволяющая оценить коммуникативную совместимость множества потенциальных научно-исследовательских коллективов на основе обработки естественной информации, генерируемой потенциальными участниками в социальных сетях.

Ключевые слова: Естественная информация, совместимость научно-исследовательского коллектива, человеческий капитал, тональность, информационная среда.

Процесс управления человеческим капиталом научно-исследовательского коллектива, под влиянием процесса цифровизации, при сохранении базисного целеполагания, трансформируется с инструментально-аналитической точки зрения. При этом, данная трансформация дифференцирована в соответствии с уровнем управления. Данная дифференциация проявляется как в не единообразном снижении трудоемкости данного процесса, так и в разнородном векторе самой трансформации. В частности, на уровне топ-менеджмента, целесообразно общее сохранение принципов управления, и лишь частичное инструментальное оснащение процесса принятия управленческих решений. Данный факт в первую очередь обусловлен значительно большим удельным весом эксперт- ной компоненты в вероятности успешности принимаемого управленческого решения, что в свою очередь обусловлено большей ценой последствий от неэффективных управленческих решений. На низовом уровне управления целесообразность максимизации инструментального оснащения процесса принятия управленческих решений также нецелесообразна. Данный факт обусловлен относительно незначительной стоимостью относительно неэффективных управленческих решений для предприятия. Следовательно, наиболее значимой в рамках цифровой трансформации и инструментальноаналитического оснащения процесса принятия управленческих решений является средний уровень управления. Также, надо отметить, что перспективность данной трансформации 
экспоненциально возрастает для проектных научно-исследовательских коллективов. В качестве универсальной задачи, можно привести формирование научно-исследовательского коллектива для выполнения проектной деятельности, имеющей прогнозируемый или условнопрогнозируемый инновационный результат и осознаваемую ограниченную продолжительность. Подобные научно-исследовательские коллективы в рамках существующей классической методологии управления формируются согласно принципу нормативного замещения необходимых для успешной реализации проекта компетенций сотрудников. Согласно данному принципу, подобная научно-исследовательская группа формируется согласно профессиональным компетенциям, которые необходимы для успешной реализации проекта и достижения поставленного инновационного результата в кратчайшие или нормативно-установленные сроки. Таким образом, данный принцип определяет необходимость максимизации физических, профессиональных, интеллектуальных и креативных качеств представителей научноисследовательского коллектива. Однако, подобный подход не предполагает учета психологической, психографической, социальной, культурной, духовной и иной совместимости членов рабочей группы. Безусловно, опытный и эффективный управленец в состоянии учесть данные факторы и сформировать максимально совместимый коллектив. Однако, данные решения, в рамках классической методологии управления, носят исключительно экспертный характер, что значительно повышает субъективность принимаемых решений и повышает ценность конкретного представителя менеджмента для предприятия. Учет данных факторов можно системно универсализировать и представить в виде математической модели, основой которой выступает параметр совместимости научноисследовательского коллектива. Под совместимостью научно-исследовательского коллектива следует понимать потенциальное приращение характеристик генерации полезного результата, за счет коммуникативной оптимизации. Следует предположить, что отсутствие коммуникативных барьеров, а также оптимальный уровень социальной, психологической и иной совместимости позволит значительно ускорить процесс генерации полезного результата, за счет отсутствия необходимости переформирования ком- муникативных паттернов, а также общей эмоциональной возвышенности.

Информационным базисом данной модели может выступать естественная информация, генерируемая потенциальными членами коллектива в социальных сетях. Данный выбор обусловлен двумя свойствами данной информации:

1. Данная информация генерируется потенциальными членами научно-исследовательского коллектива вне осмысления возможности ее использования для анализа третьими лицами и последующего принятия управленческих решений на ее основе. Данный факт определяет ее максимальную объективность.

2. Данная информация является общедоступной и не предполагает создания дополнительной трудоемкости по ее формированию.

Однако, несмотря на вышеперечисленные уникальные положительные свойства, данная информация обладает одним глобальным недостатком - она представлена в естественной форме и изначально не пригодна для машинного анализа и статистической обработки. Данный факт определяет необходимость ее первичного декодирования и последующего анализа.

Одним из ключевых свойств, принимаемых в рамках инновационной среды университета управленческих решений, является необходимая системность. При этом используемая типовая кадровая информация заменяется на естественную информацию. Следовательно, для целей оценки совместимости научноисследовательского коллектива целесообразно ввести максимизируемый критерий, в соответствии с которым будет проводиться его системная оценка. В качестве данного критерия предлагается параметр совместимости научноисследовательского коллектива. Концептуальная методика формирования максимально совместимого научно-исследовательского коллектива на основе обработки естественной информации представлена на рисунке 1.

Инструментальное оснащение процесса оценки в условиях цифровой трансформации предполагает автоматизацию каждого из этапов сформированной методики. Для целей данной автоматизации наиболее целесообразно использовать высокоуровневый язык программирования Python 3. Данный выбор обусловлен в первую очередь его универсальностью для решения каждой из задач приведенной методики, что в свою очередь обеспечивается значитель- 


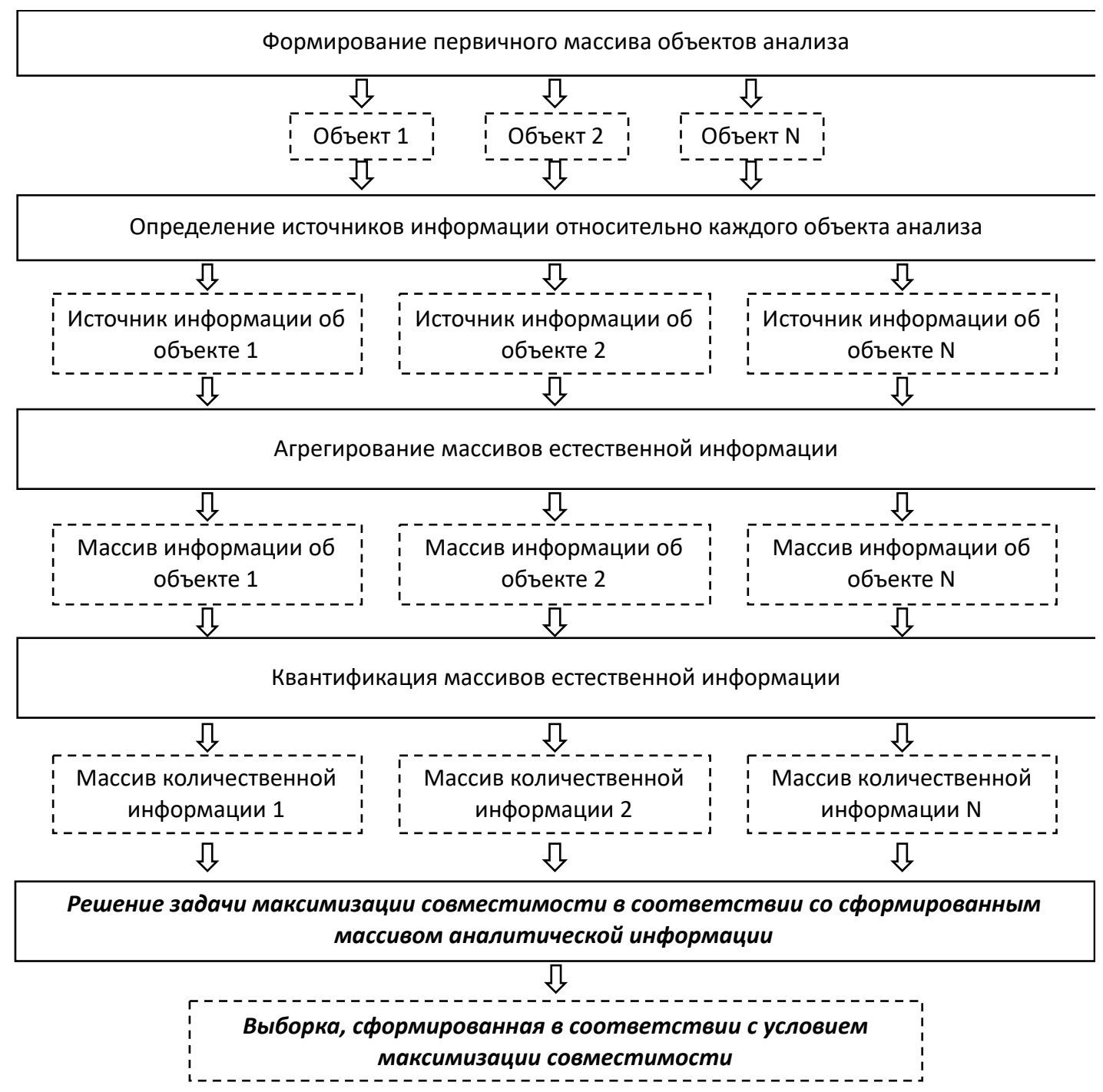

Рисунок 1. Методика формирования максимально совместимого научноисследовательского коллектива на основе обработки естественной информации

ным количеством пользовательских библиотек, во многом автоматизирующих множество промежуточных и вспомогательных алгоритмов.

Рассмотрим последовательно каждый из приведенных в описанной выше методики этапов. На первом этапе необходимо формировать первичный массив объектов анализа. Предполагается, что университет ограничивает свой выбор исключительно своими ППС и НПР, а иная совокупность кадров ограничивается своей предельной численностью. Таким образом, предполагается, что первый этап изначально включает в себя ограниченное число потенциальных членов научно-исследовательского коллектива, отобранных посредствам классической методо- логии, или сформированная под воздействием естественных факторов (таких как списочная численность персонала).

В рамках второго этапа предполагается определение источников информации относительно каждого объекта анализа. Фактически, данный этап предполагает выбор соответствующей социальной сети. Так как данная методика разрабатывается в первую очередь для университетов Российской Федерации, стоит отметить, что наиболее распространенной социальной сетью в данной стране является vk.com. Данная социальная сеть является крайне популярной не территории СНГ, во многом дублируя функционал Facebook. Также, как и Facebook, данная 
социальная сеть содержит такие элементы как «информационная лента», посредствам которой пользователи в состоянии обмениваться публичной информацией, а также выражать свое отношение к какой-либо информации посредствам лайков и репостов. Профили пользователей данной социальной сети содержат информации относительно их образования, возраста, увлечений, а также иных демографических, географических и психографических характеристик. Однако, в отличии от Facebook, vk.com обладает открытым API, что значительно повышает аналитические возможности. Таким образом, в рамках разрабатываемого методического инструмента, приведенная методика детализируется для социальных профилей анализируемых объектов в социальной сети vk.com.

На третьем этапе описанной методики производится агрегирование естественной информации о исследуемых объектам. Объем и тип исследуемой информации ограничен содержанием социальных профилей. Ранее приводился перечень первичной информации, содержащийся в рамках социальных профилей пользователей. Для определения конкретного вида информации, необходимо определиться с исследуемыми характеристиками. В первую очередь следует исключить географическую и демографическую информацию, а именно: пол, возраст, место рождения и т.д. Данное исключение обусловлено низкой информативностью приведённых данных для целей формирования ограничений. Наличие коммуникативных барьеров между уроженцами различных провинций или людьми разного возраста неизбежны в контексте непрофессионального взаимодействия. Так как в рамках научно-исследовательского коллектива предполагается исключительно профессиональная коммуникация, необходимо определить характеристики, которые могут указывать на естественные коммуникативные барьеры в рамках профессионального взаимодействия. Также, надо отметить, что учет демографических и географических характеристик подразумевает множество условных исключений, учет которых подразумевает значительное усложнение рассматриваемой методики, либо интеграцию систем экспертной оценки, что значительно повышает трудоемкость и стоимость реализации методики. Следовательно, необходимо выделить универсальные характеристи- ки процесса коммуникации. В рамках данного исследования, категориальным базисом для определения данных характеристик выступает лингвистика, так как источником проявления коммуникативных барьеров является именно процесс коммуникации, содержание которого во многом исследуется именно лингвистикой. Так как источником информации выступают профили потенциальных членов коллектива в социальных сетях, область лингвистики может быть ограничена исключительно компьютерной лингвистикой. Сам процесс профессиональной коммуникации представляет собой обмен закодированной в естественной форме информации, и направленной на достижение определенного заранее результата. В обобщенной форме, данная информация может быть охарактеризована посредствам описания двух базисных компонент:

1. Содержательная компонента. Данная компонента характеризует лексическое наполнение транслируемой естественной информации. Описание данной компоненты подразумевает не только выявление ключевых содержательных лексем и их частоты, но и несодержательных. Также, в отрыве от профессиональной коммуникации, анализ данной компоненты позволяет установить область интересов объекта исследования.

2. Эмоциональная компонента. Данная компонента характеризует эмоциональный окрас содержательной компоненты. В данном случае, наиболее эффективным инструментом описания данной компоненты является анализ тональности. Тональность - это эмоциональное отношение автора высказывания к некоторому объекту, выраженное в содержательной компоненте. Анализ тональности содержательной компоненте позволит определить как отношение субъекта к конкретному объекту, так и общие коммуникативные паттерны субъекта.

Таким образом, количественные характеристики выделенных компонент выступают в качестве предмета исследования. Объектом в данном случае могут выступать исключительно записи на странице пользователя социального профиля. Данные записи могут генерироваться как самим пользователем, так и являться репостами записей иных пользователей. Можно выдвинуть гипотезу относительно тональной и содержательной экстраполяции коммуника- 
тивных особенностей пользователя в данный массив контента. Таким образом, именно агрегированные в единообразные списки записи пользователей социальных профилей и будут выступать результатом третьего этапа методики.

На четвертом этапе методики производится квантификация агрегированной естественной информации. Как было отмечено ранее, предметом исследования выступают тональные и содержательные характеристики записей пользователей анализируемых социальных профилей. Рассмотрим первую очередь тональные характеристики. Тональность анализируемых записей может быть описана множеством характеристик. В данном случае для оценки тональности информационной среды к использованию предлагается открытая библиотека для семантического анализа средствами языка python - «Dostoevsky». Данная библиотека была сформирована на основе базы данных «RuSentiment dataset» и ее точность в соответствии с критерием F1 составляет 0,71 , что является необходимым и достаточным уровнем точности. Данная библиотека позволяет выявить такие тональные свойства текста, как нейтральность, проходной характер, негативность, позитивность и естественность языка. Каждая из приведенных характеристик измеряется от 0 до 1 . В рамках формируемого инструментария наибольшую значимость приобретают три характеристики:

1. Уровень позитивности текста записи (positive). Данная характеристика отражает уровень наличия в тексте положительного эмоционального посыла.

2. Уровень негативности текста записи (negative). Данная характеристика отражает уровень наличия в тексте отрицательного эмоционального посыла.

3. Естественность текста записи (speech). Данная характеристика отражает склонность автора текста к использованию естественной (разговорной) формы коммуникации.

Посредствам библиотеки Dostoevsky данные характеристики могут быть оценены для каждой из записей пользователя. Однако, значимость характеристик наиболее ранних записей значительно ниже в значимости характеристик более поздних записей. Данный факт обусловлен способностью личности к смене коммуникативных паттернов. Следовательно, интегральные показатели тональных характеристик анализируемых пользователей могут быть рассчитаны по- средствам следующих моделей:

$$
\begin{aligned}
T^{\text {positive }} & =\frac{\sum_{1}^{N} \frac{2 *(N-i+1)}{(N+1) * N} * t_{i}^{\text {positive }}}{N} \\
T^{\text {negative }} & =\frac{\sum_{1}^{N} \frac{2 *(N-i+1)}{(N+1) * N} * t_{i}^{\text {negative }}}{N} \\
T^{\text {speech }} & =\frac{\sum_{1}^{N} \frac{2 *(N-i+1)}{(N+1) * N} * t_{i}^{\text {speech }}}{N}
\end{aligned}
$$

Где:

1. Tpositive - интегральный коэффициент уровня позитивности распространяемого контента;

2. Tnegative - интегральный коэффициент уровня негативности распространяемого контента;

3. Tspeech - интегральный коэффициент естественности распространяемого контента;

4. $\mathrm{t}_{\mathrm{i}}$ positive - уровень позитивности i записи в анализируемом массиве;

5. $\mathrm{t}_{\mathrm{i}}$ negativ - уровень негативности $\mathrm{i}$ записи в анализируемом массиве;

6. $\mathrm{t}_{\mathrm{i}}$ speech - уровень естественности i записи в анализируемом массиве;

7. N - количество записей в анализируемом массиве;

8. і - порядковый номер анализируемой записи в массиве.

Приведенные показатели в достаточной мере могут охарактеризовать тональные свойства наиболее приемлемого для анализируемого объекта типа коммуникации. Данные показатели могут быть определены для каждого из социальных профилей и в дальнейшем должны подвергнуться процедуре анализа.

Содержательная компонента, в свою очередь может описываться в первую очередь за счет специфики и структуры лексического массива. Анализ содержательной компоненты в первую очередь направлен на определение лексических элементов, которые наиболее часто и распространенно употребляемы исследуемым объектом. Базовой категорией такого вида анализа в первую очередь может выступать лексема - слово как абстрактная единица морфологического анализа. Наиболее часто употребляемые лексемы (возможно являющиеся словосочетаниями или конкретными словоформами) могут быть 


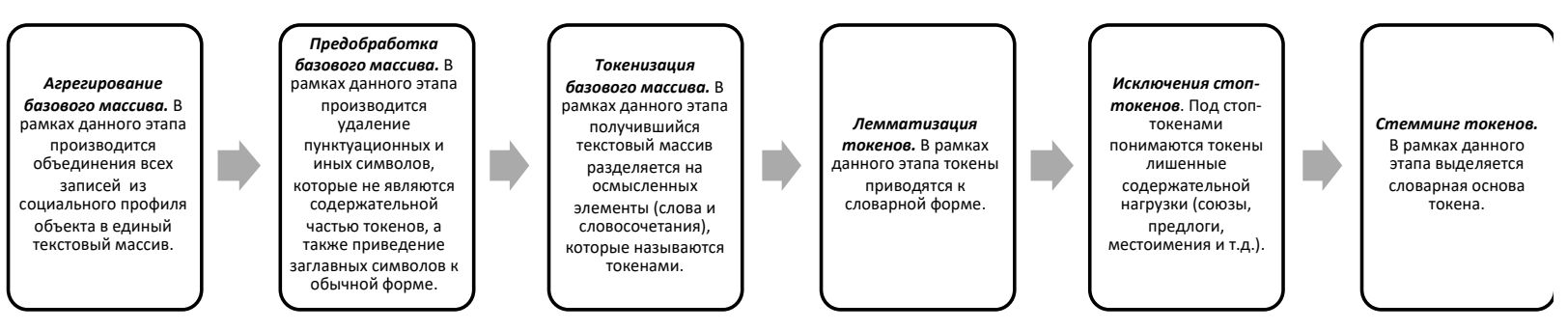

Рисунок 2. Алгоритм формирования массива токенов социального профиля

названы токенами. В качестве ключевого аналитического инструмента в данном случае предлагается использовать массивы токенов социальных профилей. В качестве квантификационного критерия (критерия важности токена) предлагается использовать частоту упоминания того или иного токена. Процедура токенизация текстового массива представлена на рисунке 2.

Полученные по результатам данного алгоритма массивы квантифицируются посредствам расчета частоты упоминания каждого токена и агрегируются в единый аналитический массив. Таким образом, анализ объектов, в рамках формируемой методики, производится на основании выделенных инструментов квантификации содержательной и эмоциональной компонент массивов текстовой информации, распространяемой анализируемыми объектами. Сформированные массивы, являются первичными массивами количественной информации.

На следующем этапе методики производится максимизация совместимости в соответствии со сформированными первичными массивами количественной информации. В соответствии с выделенным ранее определением совместимости научно-исследовательского коллектива, решение данной задачи лежит в области инструментов поиска оптимальной совокупности. Существующая в рамках данной задачи совокупность объектов, может быть разделена на два множества:

1. $\mathrm{A}_{0}$ - изначальное множество, включающее в себя всех потенциальных членов научноисследовательского коллектива;

2. $\mathrm{A}_{\mathrm{f}}$ - конечное множество, включающее в себя наиболее совместимых друг с другом членов научно-исследовательского коллектива.

Оптимизационный алгоритм решает задачу перехода от множества $\mathrm{A}_{0}$ к множеству $\mathrm{A}_{\mathrm{f}}$.При этом, оба множества необходимо разделить на единое количество профессиональных ролей, в рамках каждой из которых может быть представлено от 1 до n представителей. На рисунке
3 представлен переход от множества $\mathrm{A}_{0}$ к множеству $\mathrm{A}_{\mathrm{f}}$.

В соответствии с представленным механизмом, можно заключить, что результирующее множество Af является вариативным. Следовательно, первичным этапом определения максимально совместимого коллектива является определение всех потенциальных комбинаций данного коллектива. Далее необходимо ввести единый сравнительный критерий, который может быть определен для каждого научноисследовательского коллектива, основываться на вышеописанной специфике коммуникативных компонент, а на его сравнении должны формулироваться однозначные выводы, не требующие многомерной обработки.

В первую очередь необходимо отметить, что в рамках разрабатываемого методического инструмента удельный вес влияния эмоциональной и содержательной компонент на показатель совместимости является одинаковым. Следовательно, результирующий критерий может определяться как среднее от значений данных компонент, вследствие чего изначально данный критерий может быть разделен на две составляющие, рассматриваемые отдельно. Рассмотрим в первую очередь эмоциональную компоненту.

Для определения эмоциональной компоненты ранее было предложено три коэффициента, отражающие тональные характеристики исследуемых массивов распространяемой объектами текстовой информации. Так как каждый из вариантов потенциально совместимого коллектива содержит ограниченное число участников, по каждому из которых определены соответствующие коэффициенты, имеется возможность бинарно сопоставить каждого из их. Коммуникация в рамках подобной группы не может быть изначально нормативно ограничена, что подразумевает возможность (а скорее всего необходимость) взаимодействия каждого из участников с каждым. Следовательно, посредствам инструментов комбинаторики, могут быть составлены 


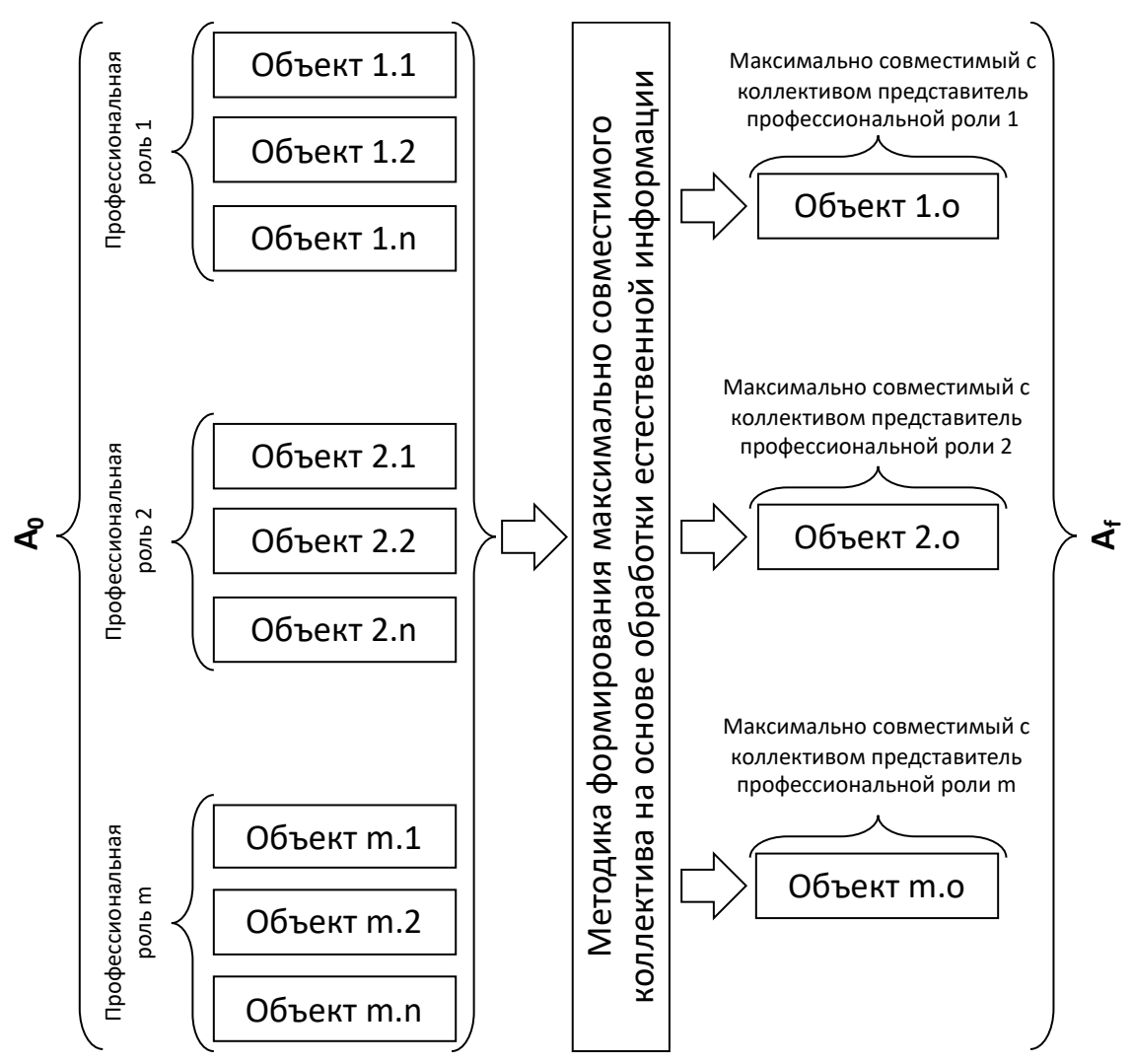

Рисунок 3. Механизм формирования наиболее совместимого научноисследовательского коллектива

пары значений коэффициентов, отражающие совместимость коммуницирующих объектов.

Стрелки в области возможных бинарных коммуникативных комбинаций отражают направления коммуникации. Совокупность соответствующих значений коэффициентов для максимально возможного числа бинарных коммуникативных комбинаций объектов формирует два сопоставимых ряда данных (ряд $e$ и ряд $u$ ). Степень подобия данных рядов будет определять уровень совместимости научноисследовательского коллектива. Одним из наи- более эффективных параметров, характеризующих данный параметр для двух рядов данных, является коэффициент корреляции Пирсона. Следовательно, при допущении, что удельный вес трех выделенных тональных характеристик является одинаковым, коэффициент тональной совместимости научно-исследовательского коллектива может быть определен следующий образом:

$$
K^{\text {T.C. }}=\frac{\left(K^{T^{\text {positive }} . C .}+K^{T^{\text {negative }} \cdot C .}+K^{T^{\text {speech }} . C .}\right)}{3}
$$

$A_{f}$

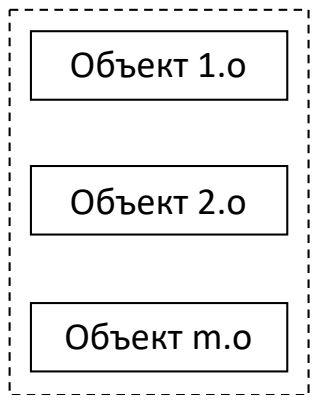

Возможные бинарные коммуникативные комбинации

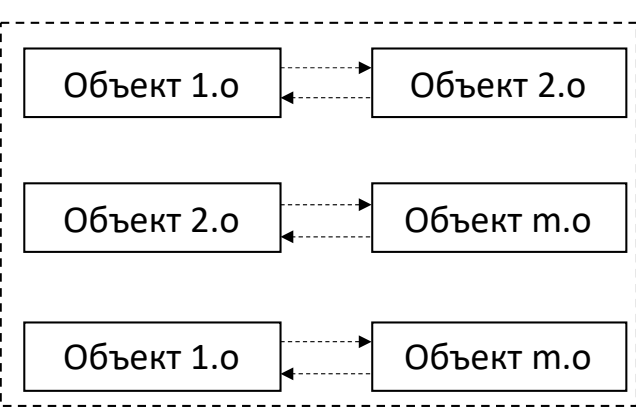

Рисунок 4. Механизм формирования бинарных коммуникативных комбинаций 
Где:

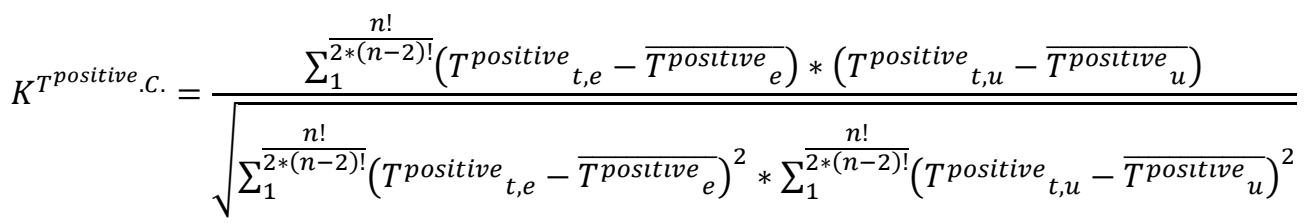

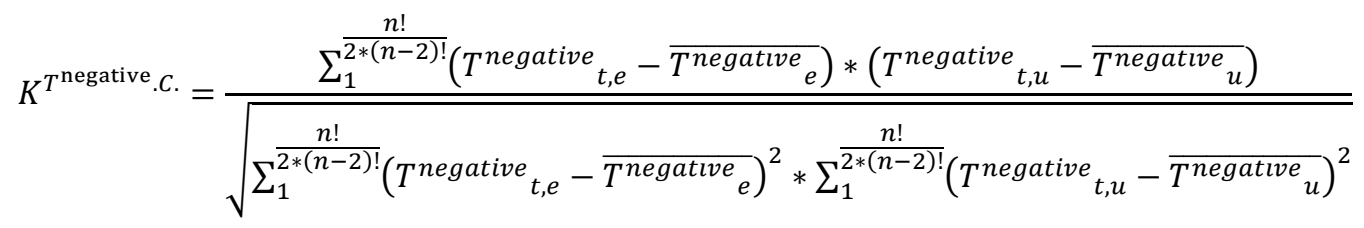

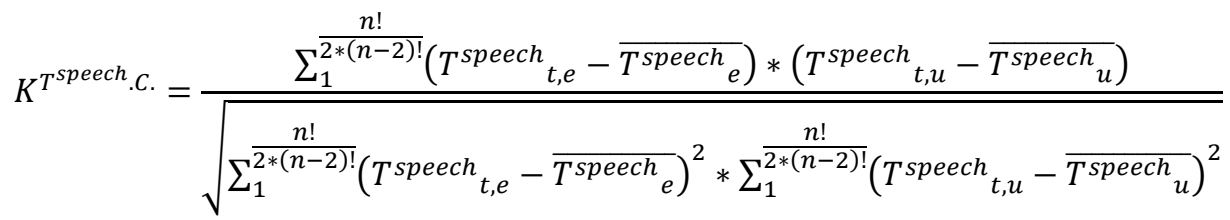

Где:

1. $\mathrm{KT}^{\text {positive.c. }}-$ коэффициент тональной совместимости научно-исследовательского коллектива в контексте уровня позитивности распространяемого контента;

2. $\mathrm{KT}^{\text {negative.c. }}$ - коэффициент тональной совместимости научно-исследовательского коллектива в контексте уровня негативности распространяемого контента;

3. $\mathrm{KT}^{\text {speech. }}$. - коэффициент тональной совместимости научно-исследовательского коллектива в контексте естественности распространяемого контента;

4. $\mathrm{n}$ - необходимое число участников научно-исследовательского коллектива, или число профессиональных ролей в коллективе;

5. Tpositive $_{\mathrm{t}, \mathrm{e}}-$ интегральный коэффициент уровня позитивности распространяемого контента объектом t в ряду $e$;

6. Tnegative $_{t, \mathrm{e}}-$ интегральный коэффициент уровня негативности распространяемого контента объектом t в ряду $е$;

7. Tspeech $_{\mathrm{t}, \mathrm{e}}-$ интегральный коэффициент уровня естественности распространяемого контента объектом t в ряду $е$;

8. Tpositive $_{t, u}-$ интегральный коэффициент уровня позитивности распространяемого контента объектом t в ряду $u$;

9. Tnegative $_{t, u}$ - интегральный коэффициент уровня негативности распространяемого контента объектом t в ряду $u$;

10. Tspeech $_{\mathrm{t}, \mathrm{u}}$ - интегральный коэффициент уровня естественности распространяемого контента объектом t в ряду $u$;

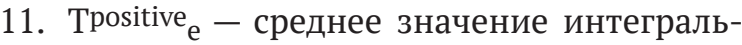
ного коэффициента уровня позитивности распространяемого контента по ряду $e$;

12. Tnegative ${ }_{\mathrm{e}}$ - среднее значение интегрального коэффициента уровня негативности распространяемого контента по ряду $е$;

13. Tspeech $_{\mathrm{e}}$ - среднее значение интегрального коэффициента естественности распространяемого контента по ряду $e$;

14. Tpositive $_{\mathrm{u}}$ - среднее значение интегрального коэффициента уровня позитивности распространяемого контента по ряду $u$;

15. Tnegative $_{u}$ - среднее значение интегрального коэффициента уровня негативности распространяемого контента по ряду $u$;

16. Tspeech $_{u}$ - среднее значение интегрального коэффициента естественности распространяемого контента по ряду $u$.

Данный коэффициент измеряется от -1 до 1 , где -1 отображает максимальную противоположность коллектива с точки зрения тональной корреляции, а 1, в свою очередь отображает предельную тональную корреляцию. Однако, максимальное значение данного коэффициента не является оптимальным значением критерия совместимости, так как при его предельных значениях возрастает риск отклонений от профессиональной деятельности, и переход к нецелевой коммуникации. В соответствии со шкалой Чедока, минимальный уровень коэффициента корре- 
ляции, отражающего высокую степень связи, является значение 0,7. В рамках текущей методике предлагается использовать, данное значение как максимально результативное, в то время как его отклонения в обе стороны не желательны. Таким образом, необходимо ввести результирующий коэффициент тональной совместимости научно-исследовательского коллектива (RT.C.), распределение которого представлено на рисунке 5.

Для целей максимально точного определения данного коэффициента в соответствии с фактическим значения коэффициента тональной совместимости научно-исследовательского коллектива предлагается использовать полиномиальную функцию пятой степени.

$$
\begin{aligned}
& R^{\text {T.C. }}=0,06+0,64 * K^{\text {T.C. }}+1,53 *\left(K^{\text {T.C. }}\right)^{2}+ \\
& 0,41 *\left(K^{\text {T.C. }}\right)^{3}-1,66 *\left(K^{\text {T.C. }}\right)^{4}-1,12 * K^{\text {T.C. }}{ }^{5}
\end{aligned}
$$

Результирующий коэффициент тональной совместимости научно-исследовательского коллектива является сравнительным параметром, в соответствии с которым можно охарактеризовать сопоставимость каждого из возможных инвариантов рабочих коллектив, сформированных на основе комбинации объектов начально- го массива, в рамках описания эмоциональной компоненты.

Далее необходимо рассмотреть содержательную компоненту, аналитическим базисом для определения которой являются массивы токенов, сформированные на основе социальных профилей. В данном случае, содержательная компонента определяет лексическую и частично тематическую совместимость потенциальных участников научно-исследовательского коллектива. Выразить данную совместимость возможно посредствам взвешенной оценки пересечения множеств токенов каждого из потенциальных участников научно-исследовательского коллектива. Удельный вес эффективно определяется частотой упоминания токена. Таким образом, в соответствии со сформированными ранее возможными бинарными коммуникативными комбинациям, для каждой из комбинаций может быть определено два множества токенов:

1. Совокупное множество токенов, включающее в себя все токены, содержащиеся в двух массивах токенов соответствующих социальных профилей.

2. Множества пересекающихся токенов, включающее в себя только те токены, которые встречаются в обоих массивах токенов соответствующих социальных профилей.

Множество пересекающихся токенов в дан-

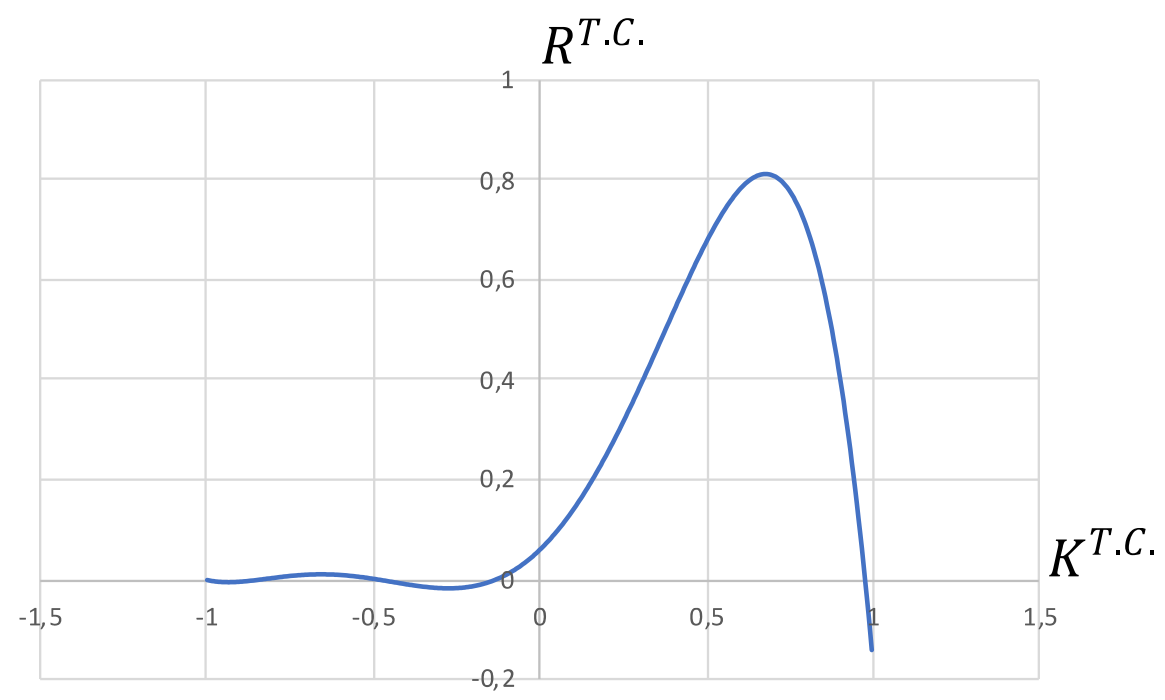

Рисунок 5. Распределение результирующего коэффициента тональной совместимости научно-исследовательского коллектива 
ном случае отражает область лексической и тематической совместимости двух потенциальных членов рабочего коллектива. Таким образом, взвешенная доля данного множества совокупном множестве токенов, отражает совместимость двух потенциальных членов научноисследовательского коллектива в соответствии с содержательной компонентой. Для каждой возможно совокупности потенциальных членов научно-исследовательского коллектива, данный параметр может быть определен в соответствии со следующей моделью:

$$
R^{C . C .}=\frac{\sum_{1}^{\frac{n !}{2 *(n-2) !}} \frac{\sum_{f_{1}}^{f_{t}} F r_{t}}{\sum_{f_{1}}^{f_{x}} F r_{x}}}{\frac{n !}{2 *(n-2) !}}
$$

Где:

1. RC.C.- результирующий коэффициент содержательной совместимости научноисследовательского коллектива;

2. $\mathrm{Fr}_{\mathrm{t}}$ - сумма частот появления пересекающегося токена $\mathrm{t}$ у обоих потенциальных участников научно-исследовательского коллектива;

3. $\mathrm{Fr}_{\mathrm{x}}$ - частота появления токена х у соответствующего потенциального участника научно-исследовательского коллектива;
4. $\mathrm{f}_{\mathrm{t}}$ - число пересекающихся токенов;

5. $\mathrm{f}_{\mathrm{x}}-$ совокупное число токенов, упоминаемых обоими потенциальными участниками научно-исследовательского коллектива.

Данный результирующий параметр измеряется от 0 до 1, а функция приращения его эффективности является линейно положительной, что определяет выбор наиболее совместимого научно-исследовательского коллектива в соответствии с максимальных значением данного параметра.

На завершающем этапе рассматриваемой методики производится анализ полученных результатов и выбор наиболее совместимой совокупности потенциальных членов научноисследовательского коллектива. Данный выбор может быть математически формализован введением коэффициента совместимости рабочего коллектива, определяемого как среднее арифметическое от результирующего коэффициента содержательной совместимости рабочего коллектива и результирующего коэффициента тональной совместимости научноисследовательского коллектива. Данный параметр измеряется от 0 до 1, определяется для каждого из вариантов потенциального научноисследовательского коллектива, а выбор производится в соответствии с универсальным правилом максимизации данного параметра.

\section{Библиографический список}

1. Родионов Д.Г. Управление информационной экономической безопасностью в системе региональной экономики // Мир экономики и права. 2012. № 1. С. 8-13.

2. Sinan Aral. Commentary-identifying social influence: A comment on opinion leadership and social contagion in new product diffusion. Marketing Science, 30(2):217-223, 2011.

3. Khim-Yong Goh, Cheng-Suang Heng, and Zhijie Lin. Social media brand community and consumer behavior: Quantifying the relative impact of user-and marketer-generated content. Information Systems Research, 24(1):88107, 2013.

4. Ruomeng Cui, Santiago Gallino, Antonio Moreno, Dennis Zhang. The Operational Value of Social Media Information. Production and Operations Management (2017), https://doi.org/doi:10.1111/poms.12707

5. Palme, J. (1972). Developments in using natural language. Computer Weekly.

6. D.P. Partridge, E. B. James. Natural Information Processing. Int. J. Man-Machine Studies (1974) 6, 205-235

7. Hajo Greif. Affording Illusions? Natural Information and the Problem of Misperception. AVANT, Vol. X, No. 3/2019 ISSN: 2082-6710 avant.edu.pl/en DOI: 10.26913/avant.2019.03.19

8. Pan, B., Crotts, J.: Theoretical models of social media, marketing implications, and future research directions. In: Sigala, M., Christou, E., Gretzel, U. (eds.) Social Media in Travel, Tourism and Hospitality: Theory, Practice and Cases, pp. 73-86. Ashgate, Surrey (2012)

9. Del Fresno García, M., Daly, A.J., Segado Sánchez-Cabezudo, S.: Identifying the new influences in the internet era: social media and social network analysis. Rev. Esp. Invest. Sociol. 153, 23-40 (2016) 
10. Ngai, E.W., Tao, S.S., Moon, K.K.: Social media research: theories, constructs, and conceptual frameworks. Int. J. Inform. Manag. 35, 33-44 (2015)

11. Pattee, H. H. Dynamic and linguistic modes of complex systems. Int. J. Genet. Syst. 1977, 3, 259-266.

12. Stanley N. Salthe. Naturalizing Information. Information 2011, 2, 417-425; doi:10.3390/info2030417 